Mots. Les langages du politique

\title{
La femme invisible. Sur l'imaginaire du pouvoir politique
}

Grégory Derville et Sylvie Pionchon

\section{(2) OpenEdition}

\section{Journals}

Édition électronique

URL : https://journals.openedition.org/mots/369

DOI : $10.4000 /$ mots.369

ISSN : 1960-6001

Éditeur

ENS Éditions

\section{Édition imprimée}

Date de publication : 1 juillet 2005

Pagination : 53-64

ISBN : 2-84788-080-1

ISSN : 0243-6450

Référence électronique

Grégory Derville et Sylvie Pionchon, "La femme invisible. Sur l'imaginaire du pouvoir politique », Mots Les langages du politique [En ligne], 78 | 2005, mis en ligne le 31 janvier 2008, consulté le 22 avril 2022. URL : http://journals.openedition.org/mots/369 ; DOI : https://doi.org/10.4000/mots.369 


\section{Grégory Derville \\ Sylvie Pionchon}

\section{La femme invisible. Sur l'imaginaire du pouvoir politique}

En dépit de l'inflexion très nette suscitée par la mise en œuvre de la loi du 6 juin 2000 sur la parité hommes/femmes en politique, le champ politique reste en France un univers très majoritairement masculin ${ }^{1}$. À la veille du référendum de mai 2005, le président de la République, le Premier ministre, les présidents de l'Assemblée nationale et du Sénat étaient des hommes; les hommes représentaient aussi $74 \%$ des ministres, $88 \%$ des député(e)s, $89 \%$ des sénateurs et sénatrices, $89 \%$ des élu(e)s aux conseils généraux (et 99 des 102 président[e]s de conseil général), $52 \%$ des élu(e)s aux conseils régionaux et 21 des 22 président(e)s de conseil régional, $89 \%$ des maires, $95 \%$ des président(e)s d'ECPI...

Comment expliquer cette sous-représentation politique des femmes? Des raisons variées peuvent être avancées. On peut ainsi invoquer des facteurs historiques (la façon dont s'est construit le champ politique français depuis 1789), des facteurs sociologiques (la moindre disponibilité des femmes pour l'engagement public), des facteurs politiques (le fonctionnement partisan) ${ }^{2}$. Mais plus fondamentalement, on constate aujourd'hui encore, dans l'esprit d'une majorité de citoyens et d'acteurs politiques des deux sexes, la prégnance de représentations sociales qui sont largement antagonistes avec l'engagement politique au féminin. Il s'agit d'abord des représentations concernant les rôles de sexe. L'idée selon laquelle c'est en priorité à la femme de s'occuper de l'espace domestique et des enfants, car c'est avant tout là qu'elle doit s'y réaliser, est loin d'avoir disparu³. Pour faire carrière en politique, les femmes doivent

1. Cet article est issu d'une thèse de science politique soutenue en 2000 par S. Pionchon à l'IEP de Grenoble (Les femmes et la politique. Éléments d'explication d'une sous-représentation), thèse elle-même synthétisée et actualisée dans un ouvrage paru en 2004 (S. Pionchon et G. Derville, Les femmes et la politique en France, Presses universitaires de Grenoble, 215 p.). Sauf indication contraire, toutes les citations émanent directement de la thèse de S. Pionchon et peuvent aussi être retrouvées dans l'ouvrage qui en est tiré.

2. Dans notre récent ouvrage, nous avons présenté une revue détaillée de ces explications (et d'autres...), avec de nombreux exemples à l'appui (S. Pionchon et G. Derville, 2004, op. cit.).

3. S. Pionchon et G. Derville, 2004, op. cit., p. 107-121.

Université Lille 2, CERAPS-CNRS, gregory.derville@wanadoo.fr 
bien davantage que les hommes pouvoir faire la preuve de leurs bons états de service familiaux, ce qui restreint l'éventail de leurs possibilités. En second lieu, le champ politique reste, on le verra, très largement pensé comme un univers viril. Pour ces deux raisons, la présence massive des femmes dans le champ politique, à parité avec les hommes, est difficilement acceptable, voire pensable, du moins à des niveaux élevés de la hiérarchie.

Ces représentations sociales s'expriment au sein du personnel politique via des discours et des slogans sexistes, parfois tenus en public (en particulier lors des campagnes électorales). En outre, elles se traduisent par une marginalisation tangible des femmes politiques: même si c'est moins net dans le contexte paritaire actuel, les partis sont en général assez réticents à leur accorder des investitures (surtout dans des circonscriptions gagnables) et des postes éminents (présidences d'assemblées, ministères pleins...). Tous ces éléments, que nous aborderons en premier lieu, incitent à poser le diagnostic d'un champ politique sexiste et travaillant à exclure les femmes, ou au moins à les reléguer dans ses zones déclassées et déclassantes. Cependant, cette explication n'est pas la principale (sans compter qu'elle est, on y reviendra, moins pertinente qu'il y a quelques années) : nous verrons dans un second temps que la réticence d'une forte proportion de dirigeants partisans, d'élus, de militants, mais aussi de citoyens, à envisager la présence massive des femmes en politique, tient en fait à des représentations plus générales très profondément ancrées dans les esprits.

Notre analyse s'appuiera largement sur une série de très longs entretiens semi-directifs (trente-deux au total) réalisés par Sylvie Pionchon auprès de dix hommes et vingt-deux femmes de tous profils sociaux, culturels, intellectuels et politiques comprenant des militant(e)s, des élu(e)s, de simples citoyen(ne)s. Ces personnes ont été invitées à s'exprimer à partir d'une consigne très large («)'aimerais que nous parlions de la politique, ce qu'elle signifie pour vous, ce que vous en pensez »), ce qui a permis de constater les thèmes, les images et le vocabulaire qui surgissent spontanément dans leur esprit lorsqu'ils ou elles réfléchissent à ce thème, mais aussi de mesurer les différences de politisation entre les enquêté(e)s en fonction de leur profil. Ces trente-deux entretiens ont été analysés de façon thématique et qualitative (tous les propos cités dans cet article en sont extraits), mais ils ont aussi été intégralement retranscrits et analysés de façon quantitative à partir d'un logiciel de lexicométrie élaboré par Dominique Labbé, ce qui est une première pour un corpus oral si vaste (345 724 mots) $)^{4}$.

4. La présentation de la méthodologie retenue pour la réalisation et l'analyse des entretiens est à lire dans S. Pionchon, Les femmes et la politique, op. cit., annexes. 


\section{Le fonctionnement sexiste du champ politique}

Pour décrire la domination masculine sur le champ politique, la sociologue Françoise Gaspard propose le concept de «fratriarcat »". Depuis 1789 et jusqu'à une période récente, les hommes ont fait de la politique entre eux. L'espace politique était alors non mixte, un peu sur le modèle du vestiaire de rugby ou de la chambrée de caserne. Les règles du jeu politique, ses pratiques, sa culture, sa langue, ont été fondées et institutionnalisées par et pour des hommes.

Ceci a conduit de nombreux auteurs à expliquer la sous-représentation des femmes dans le champ politique par la volonté des hommes politiques d'en exclure les femmes. Cette volonté tiendrait d'abord très prosaïquement au fait qu'ils refusent de partager le pouvoir avec elles. En politique, une femme en plus, c'est un homme en moins : aux yeux de ses collègues masculins, la femme politique est alors une concurrente, une intruse, une voleuse d'emploi. Qui plus est, la plupart des hommes politiques n'ont guère envie de voir des élues introduire dans l'agenda politique des revendications féministes: leur résistance à l'entrée des femmes en politique est aussi un moyen de défense contre une remise en question de la domination masculine sur l'ensemble de la société.

De nombreux éléments peuvent être cités à l'appui de cette thèse et illustrent la forte résistance du champ politique à la féminisation. En effet, les femmes y subissent des discriminations spécifiques, qui sont épargnées aux hommes, et qui ont pour résultat d'amoindrir leur légitimité et leur autorité politiques et de rendre l'exercice de l'action politique plus pénible et décourageant pour elles que pour leurs collègues masculins. Pour s'en tenir aux discours et aux représentations, les femmes font bien plus souvent que les hommes l'objet d'interrogations sur leur vie privée. Elles ont le sentiment de devoir rendre des comptes sur cette dimension de leur vie, de devoir faire la preuve de leur féminité à travers le statut d'épouse et de mère. De fait, la femme célibataire et/ou sans enfant est souvent exposée à des insinuations, des rumeurs, voire de véritables agressions, comme dans cette interpellation subie par Arlette Laguillier lors d'un passage à "L'Heure de vérité » sur Antenne 2: «N'avez-vous pas l'impression d'avoir raté votre vie de femme?» Le fait d'occuper une position d'autorité et de pouvoir est encore si peu associé à la féminité que les élues sont souvent confrontées à des situations qui les nient dans leur fonction politique. Beaucoup racontent que, lors des cérémonies officielles, les huissiers les orientent spontanément vers la rangée

5. F. Gaspard, 1995, "Des partis et des femmes», M. Riot-Sarcey éd., Démocratie et représentation, Paris, Kimé. 
des épouses, ou se plaignent de subir bien plus de désintérêt et de brouhaha que leurs collègues masculins lors de leurs prises de parole publiques... Par ailleurs, les femmes politiques sont souvent la cible d'une hostilité qui vise non seulement leur action (comme c'est aussi le cas des hommes), mais aussi leur identité féminine. Nombreuses sont celles ${ }^{6}$ qui dénoncent ainsi la grivoiserie, voire les injures sexuelles dont elles ont été les victimes, parfois du fait d'anonymes (tracts, affiches électorales taguées) ou de manifestants (alors ministre de l'Environnement, Dominique Voynet fut traitée de "salope» lors d'un salon de l'Agriculture), mais parfois aussi dans les assemblées politiques : il n'y a pas dix ans, Roselyne Bachelot affirmait que l'injure sexiste était monnaie courante au Palais Bourbon ("L'Assemblée nationale est un haut lieu du machisme et du sexisme en France, l'ambiance y est celle d'une chambrée de caserne »). Dans tous les cas, l'agression sexiste révèle combien la femme n'a pas encore, en politique, la même légitimité que l'homme, si bien qu'elle n'est pas autant protégée que lui par sa fonction - le traitement infligé à Édith Cresson lors de son accession à Matignon en 1991 est ici très éloquent...

Sans doute y a-t-il là une explication de la moindre appétence politique des femmes : elles pressentent la violence dont les femmes politiques peuvent faire l'objet, et elles ne peuvent qu'hésiter, voire être dissuadées, au moment de se lancer dans un univers qui est, pour leur sexe, particulièrement hostile et anxiogène. Quant à celles qui s'engagent malgré tout, le sexisme peut dans certains cas les décourager de faire carrière dans un univers où elles ont le sentiment de prendre plus de coups que leurs collègues masculins. Par un lapsus particulièrement révélateur, une militante rencontrée par S. Pionchon laisse d'ailleurs entrevoir comment elles peuvent intérioriser la pression particulière qui pèse sur elles: elle lâche en effet un «il faut qu'on se prouve», avant de reprendre (« il faut prouver notre compétence »). Ce témoignage n'est pas isolé : toutes les militantes et les élues rencontrées dénoncent le fonctionnement misogyne du jeu politique et disent faire l'objet de discriminations du simple fait de leur sexe. S'agit-il d'un discours de rationalisation destiné à masquer une incapacité personnelle, ou est-ce un compte rendu exact de la réalité? Après tout, la politique est cruelle pour les hommes aussi... Quoiqu'il en soit, le fait que toutes ces femmes tiennent un discours de ce type montre bien que la présence féminine en politique continue à être vécue et décrite comme une transgression. À travers leur sentiment d'illégitimité, elles laissent entendre qu'elles sont désignées (ou du moins qu'elles se vivent) comme des usurpatrices qui viennent dérober cet attribut masculin qu'est le pouvoir politique.

Pour conclure sur la résistance du champ politique à la féminisation, il faut enfin insister sur le sexisme du fonctionnement partisan. Les femmes

6. Voir par exemple Elisabeth Guigou (1997, Être femme en politique, Paris, Plon), ou Frédérique Bredin (1997, Députée, journal de bord, Paris, Fayard). 
politiques (et celles qui veulent le devenir) sont d'abord défavorisées par le fait que les hommes sont très largement majoritaires dans les partis, notamment au niveau des postes dirigeants et dans les commissions d'investiture. En outre, les hommes ont davantage de chances d'être choisis comme candidats car ils sont objectivement mieux dotés que les femmes (ils sont plus souvent sortants et/ou cumulards), mais aussi parce que les dirigeants partisans (et plus encore les militants de base) ont tendance à penser qu'un homme est par nature plus apte à faire campagne et à assumer un mandat: il est censé disposer d'une autorité, d'une légitimité, d'une disponibilité et d'une résistance qui font a priori défaut aux femmes ${ }^{7}$. Bref, la sous-représentation politique des femmes est très largement imputable à l'antiféminisme des dirigeants partisans et de leurs militants: les partis fonctionnent aujourd'hui encore comme des «lieux de confiscation du pouvoir au profit des hommes $"^{8}$.

Il convient toutefois de nuancer cette analyse. Tout d'abord, elle vaut bien moins aujourd'hui qu'il y a seulement vingt ans. Si les femmes restent minoritaires dans le champ politique, surtout à ses échelons les plus prestigieux, leur proportion est quand même en très forte hausse, en particulier depuis le vote de la loi sur la parité. Du fait de cette féminisation rapide du personnel politique, ainsi que de l'action de mouvements féministes comme les Chiennes de garde, les discours et les pratiques sexistes en politique sont de plus en plus susceptibles de susciter la réprobation et de disqualifier leurs auteurs (ce qui ne signifie bien sûr pas qu'il n'y en ait plus, le sexisme pouvant se recomposer sous des formes moins visibles et vulgaires...). Mieux encore, le fait d'être une femme en politique peut parfois être un avantage. Dans un contexte paritaire où les partis ont besoin de trouver de nouvelles figures féminines, les femmes peuvent constituer des "produits d'appel» intéressants, et en tous cas il devient très risqué de les marginaliser de façon trop voyante (voir le tollé suscité par Alain Juppé lorsqu'il a exclu de son gouvernement celles que l'on appelait alors les «Juppettes»). On peut expliquer ainsi une certaine surenchère dans l'affichage de l'identité féminine de la part de quelques candidates lors des récentes campagnes électorales ${ }^{9} \ldots$ avec néanmoins le risque d'enfermement dans un «éternel féminin » largement incompatible avec l'exercice de l'action politique au jour le jour ${ }^{10}$ !

7. Sur les procédures de sélection des candidats, voir S. Pionchon et G. Derville, 2004, op. cit., p. $74-81$.

8. M. Sineau, 1988, Des femmes en politique, Economica, p. 3.

9. Voir par exemple S. Bonnafous, 2003, "Femme politique": une question de genre?», Réseaux, $\mathrm{n}^{0} 120$.

10. C. Achin et M. Paoletti ont bien montré comment le genre féminin a été constitué en ressource par de nombreuses candidates aux élections municipales de 2001... avant de redevenir, notamment au moment de la constitution des exécutifs, un stigmate (C. Achin et M. Paoletti, 2002, "Le "salto" du stigmate. Genre et construction de liste aux municipales de 2001 ", Politix, nº 6o, p. 33-54). 
En second lieu, l'indéniable résistance du champ politique à la féminisation ne signifie en rien qu'il existerait en son sein une sorte de complot des hommes dans le but d'en bannir les femmes. À vrai dire, imputer la sousreprésentation politique des femmes à la seule mauvaise volonté des hommes politiques nous parait extrêmement réducteur, surtout dans le contexte actuel. Si cette mauvaise volonté existe bel et bien chez certains (mais pas chez tous), elle s'appuie en fait sur des représentations qui sont très largement diffusées au sein de la société toute entière (c'est particulièrement vrai pour les représentations $d u$ bon candidat). C'est sans doute ici que la résistance à la féminisation du champ politique est et restera la plus forte, d'autant plus qu'elle est profondément ancrée dans les esprits, constitutive des identités de sexe et dissimulée derrière tout un discours selon lequel l'égalité entre les hommes et les femmes serait désormais «acquise».

\section{La force des représentations sociales}

Si les femmes sont très handicapées dans leur accès au champ politique, ce n'est pas tant du fait de la résistance des hommes politiques qu'en raison de causes à la fois plus profondes, plus structurelles et plus durables: aujourd'hui encore, les représentations sociales font obstacle à l'entrée des femmes en politique et ont pris le relais de l'exclusion expresse. Les représentations des identités féminine et masculine et des rapports entre les hommes et les femmes, et plus encore les représentations de la politique (conçue comme une sphère et une activité viriles), concourent à inscrire dans les esprits l'idée d'une illégitimité politique des femmes.

Encore aujourd'hui, être un homme et détenir le pouvoir sont indissociables dans les esprits. Comme le montrent les anthropologues Françoise Héritier ou Georges Balandier, ce schéma patriarcal est quasiment universel. Dans presque toutes les sociétés, le pouvoir est lié à l'exercice de la puissance virile. Dans les royautés africaines traditionnelles, par exemple, les mythes qui justifient la possession du pouvoir politique comportent très souvent une séquence relative à une prouesse sexuelle accomplie avec le phallus. Sous l'Ancien Régime en France, la loi salique excluait les femmes de la succession. À la Révolution, notre culture politique démocratique s'est construite sur des normes viriles, par exemple à travers l'association entre la citoyenneté et le droit de porter les armes. Il en reste encore des traces dans le discours politique: "Les villes, c'est comme les femmes, il ne suffit pas de les séduire, il faut savoir les prendre»(R. Barre); une campagne présidentielle nécessite une «énergie virile» (L. Jospin)...

La conception selon laquelle la politique est une activité virile par excellence se retrouve aussi dans l'esprit de nombreux citoyens et citoyennes. II 
n'est qu'à voir les réponses aux sondages portant sur l'exercice des responsabilités politiques : plus un poste est auréolé de prestige et d'autorité, et plus la perspective de le voir confié à une femme suscite des réticences ${ }^{11}$. L'analyse des entretiens réalisés par S. Pionchon confirme que dans les mentalités, le pouvoir politique continue à être spontanément associé au masculin. Les femmes sont bien moins présentes que les hommes dans le discours des enquêté(e)s, alors même que l'entretien portait en grande partie sur leur présence en politique et que plusieurs relances les ont expressément invités à en parler : parmi les personnalités citées, 80 \% sont des hommes. Par ailleurs, la féminisation des dénominations des fonctions politiques, c'est-à-dire la propension à les mentionner au féminin («la ministre »), est assez rare: $35 \%$ dans le corpus des enquêtées, et seulement $12 \%$ dans celui des enquêtés. Qui plus est, les fonctions qui sont les plus facilement évoquées au féminin sont à la fois celles qui sont les plus féminisées, les moins prestigieuses et les moins stratégiques dans une carrière politique : candidate $(66 \%)$, conseillère municipale ( $47 \%$ ), adjointe ( $46 \%$ ) ; en revanche, le titulaire de la présidence de la République n'est quasiment pas mentionné au féminin (9\%). Notons enfin que les enquêté(e)s utilisent 11 fois moins la formule «femme politique » que la formule «homme politique».

L'impression d'une invisibilité politique des femmes est confirmée par l'analyse de quelques univers lexicaux de notre corpus d'entretiens comme, par exemple, celui de l'adjectif «politique». Cet univers lexical comprend en effet le mot «homme», mais pas le mot «femme». Pour les enquêtés, parler des acteurs politiques, c'est d'abord et surtout parler de l'homme politique. Et le genre masculin dont il s'agit ici n'est pas « universel » ou «neutre » mais restrictif, en ce sens qu'il ne désigne qu'une seule moitié de l'humanité. Dans l'esprit des enquêté(e)s, les « hommes politiques » sont bien des individus mâles, comme on peut s'en apercevoir à travers certains «détails» éloquents: par exemple, plusieurs enquêté(e)s critiquent le manque de représentativité des dirigeant(e)s politiques en évoquant le "costume trois pièces », "l'attachécase » ou la "cravate», soulignant que ce n'est pas ce que porte le «citoyen lambda ». Ces images restreignent clairement l'exercice du jeu politique à un seul sexe, sans même le nommer. Autre exemple, une étudiante désigne tout au long de son entretien les femmes politiques par le pronom «ils » : leur participation au jeu politique semble conserver pour elle un caractère étrange ou anormal. De même, alors que l'enquêtrice vient de citer quelques noms de femmes politiques et de prononcer le terme «femmes politiques», ce jeune étudiant souligne qu'à sa connaissance ce terme n'existe pas («)'ai jamais

11. Voir un sondage BVA de janvier 1989: invités à dire s’ils pensent qu'une femme peut exercer aussi bien qu'un homme la présidence de la République, seuls 54 \% répondent par l'affirmative. Plus récemment, $48 \%$ des sondés ont estimé que «le candidat idéal à l'élection présidentielle» devrait plutôt être un homme (CSA, janvier 2002). 
entendu le mot "femme politique". Je sais pas comment on appelle une femme qui fait de la politique... Enfin on dit une femme qui fait de la politique... [On les appelle] pas des femmes politiques. Non, on les appelle pas. On dit rien. Voilà, un homme politique c'est quelqu'un qui fait de la politique... »).

En somme, le recours quasi exclusif au genre masculin entérine (et renforce) l'illégitimité politique des femmes, le partage inégal du pouvoir politique entre les sexes, et finalement la domination masculine sur le champ politique. Ceci n'est bien sûr pas propre à cet espace social. C'est dans toute la société que le langage reflète (autant qu'il contribue à les perpétuer) l'état des rapports de sexe et l'appropriation du pouvoir par les hommes. La domination masculine est inscrite au cœur de la langue (donc de notre inconscient individuel et collectif). En effet, la suprématie grammaticale du masculin générique concourt à construire une identité féminine dévalorisée et portée à l'effacement. Les femmes ont plus de mal que les hommes à se penser et à s'affirmer comme sujets, car elles ne peuvent se faire entendre qu'en utilisant un langage qui, sous bien des aspects, les rabaisse ou les nie. À force d'entendre que «le masculin l'emporte sur le féminin » et représente à lui seul les deux sexes, à force de constater que le masculin est toujours associé aux fonctions sociales prestigieuses et valorisées, elles intériorisent le sentiment de leur impuissance et de leur infériorité. À travers la langue, la société les incite au silence et à la transparence, et cette injonction active chez elles des blocages et des conduites d'effacement par lesquelles elles s'excluent des lieux où la langue ne les représente pas... comme le champ politique ${ }^{12}$. Les hommes sont ici à l'aise avec un langage qui exprime leur identité de sexe et conforte leur puissance, mais les femmes, étant le plus souvent désignées au masculin, sont confrontées à l'idée de la transgression, de l'usurpation, de l'illégitimité.

La langue est bien politique: elle façonne les consciences et la réalité sociale et elle participe à l'inscription dans les esprits de la division sexuée du travail et de la hiérarchie entre les sexes. Dans le monde du travail, plus un métier est prestigieux et plus la résistance à la féminisation est active : de rares femmes travaillent dans un univers d'hommes, mais il n'y a pas « les mots pour les dire », et elles sont donc rendues invisibles. Ces métiers résistent aussi bien à l'entrée des femmes dans leur univers qu'à leur désignation linguistique. Il en va de même en politique, et les mots utilisés par les enquêté(e)s pour désigner les femmes politiques n'ont rien d'anodin : ils contribuent à occulter les femmes derrière le genre masculin et à entériner l'idée que l'activité politique est fondamentalement masculine. On peut parler ici d'une domination linguistique : le champ politique est un univers d'hommes non seulement parce qu'il est peuplé d'hommes, mais aussi parce qu'il est symbolisé par les hommes.

12. Voir P. Niedzwiecki, 1993, "Femmes et langage », Cahier Femmes d'Europe, nº 40, p. 4-97. 
Dans le discours médiatique aussi, il est très fréquent que l'existence même des femmes politiques soit omise, ou même occultée. Ainsi Michèle Cotta écrit-elle dans Le Point (19 décembre 1977) que «la vie familiale au sens classique du terme: femme, enfants, distractions, loisirs, est tout à fait compromise par l'exercice du mandat électoral». Par l'emploi de cette formule sexuée («conjoint» aurait été plus pertinent), le discours entérine ici l'anormalité de la présence féminine en politique. Même chose, plus récemment, dans un reportage de l'émission de M6, «Culture pub », consacré à la communication électorale, où il était dit que les «hommes politiques » en campagne «montrent leur vie privée, leurs loisirs, leur femme» (12 octobre 2003). Enfin, l'invisibilité des femmes politiques est parfois affirmée au niveau institutionnel, comme lorsqu'en 2003, le service d'information du gouvernement diffuse à l'ensemble des ministères un « Schéma directeur de la communication gouvernementale» dans lequel il est conseillé de présenter chaque membre du gouvernement comme un «bon père de famille »...

Dans les mentalités, un consensus relatif semble donc se dégager: le pouvoir politique est plus ou moins réservé aux hommes, du moins pour les responsabilités les plus élevées. D’ailleurs, si on invite des enquêté(e)s à réagir à la présence des femmes en politique, on voit alors surgir une foule de représentations très stéréotypées de la féminité, qui sont pour ces femmes autant de critères de décrédibilisation.

Tout d'abord, presque tou(te)s les enquêté(e)s s'interrogent spontanément sur leur vie privée, ce qu'ils (elles) ne font jamais quand ils parlent de la «classe politique» ou des «hommes politiques» en général. Ceci confirme que l'emploi de ces deux formules ne concerne en fait dans leur esprit que les mâles. Tant que la réflexion concerne une classe politique indéterminée, elle se borne à l'engagement public des personnes. En revanche, dès qu'elle se focalise sur les femmes politiques, elle introduit le thème de la famille, soit de façon simplement curieuse (ont-elles le temps de s'occuper de leurs enfants, de leurs conjoints ?), soit de façon plus normative (leur action politique étant vue par certains comme incompatible avec l'équilibre de leur sphère familiale). En ce sens, le statut des femmes politiques est très particulier: elles sont sommées de correspondre avant tout au modèle de la bonne épouse et de la bonne mère... et ceci peut très bien refroidir les ambitions chez celles qui sont ainsi interpelées en réunion publique: "Tes gosses, qui est-ce qui va te les garder? [...] Qui va faire la soupe de ton mari ?»

Par ailleurs, la façon dont la plupart des enquêté(e)s parlent des femmes politiques montre bien qu'à leurs yeux, elles ne sont admises qu'à la condition de respecter les codes sociaux de la féminité. Une femme n'a de légitimité à faire de la politique que si elle « ressemble à une femme » (y compris en tant qu'objet sexuel) et si elle y met en pratique les qualités «typiquement féminines» que sont la douceur, la sensibilité, le sens de l'écoute, le dévouement, etc. En ce 
sens, la politique au féminin apparait (ou plutôt doit apparaitre) comme un prolongement de qualités affectives et émotionnelles que les femmes sont censées mettre déjà en œuvre dans la sphère privée. L'ambition politique au féminin doit ainsi être contenue dans des limites étroites que les enquêté(e)s ne cessent de rappeler: le narcissisme, l'égoïsme et le cynisme, ces «travers» que l'on condamne chez les hommes, mais qui contribuent à asseoir leur autorité, sont interdits aux femmes.

Il est vrai que les stéréotypes de la féminité font depuis une dizaine d'années, principalement depuis la loi du 6 juin 2000 sur la parité, l'objet d'une très forte valorisation sociale et médiatique : les femmes sont désormais célébrées en politique... mais c'est toujours en tant que femmes qu'on les y attend, pour y apporter leur « touche féminine», pour y faire parler leur «bon sens», leur «sensibilité » ou leur «proximité avec les problèmes quotidiens des Français». Même si elles sont plus facilement admises qu'avant, les femmes politiques restent ainsi en quelque sorte piégées par leur féminité (d'où les tentatives de nombre d'entre elles pour gommer leur appartenance à ce sexe dévalorisé et infériorisé).

Qui plus est, bien que socialement valorisés, les stéréotypes de la féminité peuvent être retournés pour reléguer les femmes vers les secteurs les plus déclassés du champ politique (le social notamment), voire pour leur dénier toute légitimité à exercer le pouvoir au motif qu'elles manquent de hauteur de vue, de sang-froid, de capacité de synthèse, d'esprit de décision. De fait, certain(e)s enquêté(e)s, en particulier des femmes, se montrent réservé(e)s ou même critiques à l'égard de la participation politique des femmes. À leurs yeux, la femme de pouvoir est une usurpatrice car elle transgresse deux principes essentiels, la division sexuelle du travail et la hiérarchie des sexes. Ils ou elles estiment d'abord que les qualités propres à leur sexe (la sensibilité, le sens de l'écoute...) rendent les femmes incapables de faire face à la dureté du jeu politique... sauf à tellement forcer leur caractère que cela devient contrenature. Alors qu'ils (elles) admettent et même valorisent le fait qu'un homme politique soit autoritaire, combattif ou agressif, ils (elles) fustigent les femmes politiques qui adoptent un comportement de ce genre (aux deux sens du mot). La femme de pouvoir, autoritaire et dure, est en fait anormale, voire monstrueuse. Ne collant plus aux clichés de la féminité, son identité de sexe est mise en question: «Les femmes qui sont en politique, elles ont fait un métier qui était réservé aux hommes, donc elles ont pris le machin des hommes... Je veux pas être méchante, mais est-ce que ce sont encore des femmes?»

Dès lors, les femmes ne semblent pas pouvoir approcher le pouvoir politique comme des individus neutres. Elles sont enfermées dans la particularité de leur sexe, ce qui les empêche de désirer et d'endosser le pouvoir avec la même légitimité que les hommes. Les représentations des enquêté(e)s manifestent donc bien le rapport problématique et ambigu qu'entretiennent à leurs 
yeux les femmes avec le pouvoir: elles peuvent avoir de l'ambition, mais seulement pour la mettre au service de la collectivité; elles peuvent assumer des responsabilités politiques, mais seulement pour y révéler leur «nature» féminine. Ainsi, ces représentations stéréotypées du pouvoir au féminin jouent un rôle fondamental dans l'éviction des femmes du politique, tant il est vrai qu'elles sont largement partagées non seulement par les citoyen(ne)s-électeurs et électrices, mais aussi par les militant(e)s, les dirigeant(e)s des partis, les professionnel(le)s des médias et du conseil en marketing politique, c'està-dire par tous ceux et toutes celles qui, chacun(e) à son niveau, contribuent à la gestion des carrières politiques.

Il faudrait encore (mais ce serait l'objet d'un autre texte) évoquer ce qui, à nos yeux, est désormais le facteur explicatif essentiel de la sous-représentation politique des femmes et de leur relégation dans les zones déclassées et déclassantes de l'espace politique: les représentations que nous avons brièvement présentées sont intériorisées avec une force toute particulière par les femmes, si bien qu'elles développent face au pouvoir politique des complexes d'infériorité, des conduites d'évitement et de mise en échec que l'on rencontre bien moins souvent chez les hommes, y compris à niveau socioculturel inférieur (PCS, niveau de diplôme...).

Pour bien comprendre, il ne faut pas hésiter à remonter très loin en arrière. Nous avons tenté de montrer ailleurs comment la socialisation reçue, dans la prime enfance, par les individus des deux sexes, les amène à envisager leur vie, à l'âge adulte, de façons sensiblement différentes ${ }^{13}$. Alors que pour les hommes, le travail et l'insertion dans l'espace public sont au fondement de leur identité, c'est l'ancrage dans la famille et la conformation au rôle de mère qui, pour les femmes, tendent à occuper cette fonction. De ce fait, les hommes et les femmes tendent à structurer leur personnalité autour de fantasmes d'autoréalisation très différents ${ }^{14}$. Toutes les enquêtées rencontrées par S. Pionchon ont manifesté un investissement affectif très fort sur leur famille, et toutes ont dit à quel point celle-ci est tellement au cœur de leur vie qu'elles n'envisagent pas de se lancer à corps perdu dans une activité publique, du moins tant que leurs enfants ne seront pas grands: elles ont fait clairement le lien entre leur ancrage familial et leur non-engagement politique. Quant aux militantes, toutes ont fait part de leurs doutes et de leur sentiment de culpabilité. En revanche, les hommes rencontrés ont très rarement évoqué leur vie

13. S. Pionchon et G. Derville, 2004, op. cit., chap. 5 .

14. Nous sommes bien sûr conscients que les choses sont, dans la réalité des situations personnelles, bien plus subtiles, mais à nos yeux, l'existence indéniable de cas particuliers (femmes hyperactives et sans enfant, hommes en congé parental...) ne suffit pas pour infirmer la tendance générale, même s’ils sont plus nombreux qu'il y a quelques décennies. 
familiale, et jamais avec culpabilité, y compris pour les militants très engagés qui, par ailleurs, se sont demandé «comment font les femmes politiques» pour supporter d'être à ce point éloignées de leurs enfants. On peut résumer ce point en disant que les modèles de réalisation qui leur sont socialement prescrits poussent les femmes à trouver davantage de gratification et d'épanouissement dans l'endossement des rôles familiaux et maternels.

Par ailleurs, on peut penser que la socialisation anticompétitive qui leur est souvent prodiguée (en tous cas davantage qu'aux garçons) fait que les femmes, devenues adultes, présentent moins souvent les traits de personnalité indispensables pour s'affirmer dans un univers aussi concurrentiel et âpre que le jeu politique - ce que l'on appelle en psychosociologie l'assertivité et la capacité de dominance. De ce fait, elles tendent à anticiper avec plus d'appréhension que les hommes les difficultés objectives de la course au pouvoir (sacrifice de la vie privée, machiavélisme, stress, solitude affective...), d'autant plus qu'elles imaginent (avec certaines raisons, on l'a vu) que le sort qui est réservé à leur sexe est ici tout particulièrement difficile. Cette vie est-elle si enviable? Les bénéfices que l'on peut en retirer (honneurs, sentiment de puissance...) en valent-ils la peine, justifient-ils le cout de l'engagement? Là encore, voici des questions que bon nombre d'enquêtées ont spontanément et longuement évoquées pour expliquer leur réticence à faire de la politique, ce qui n'a été le cas d'aucun enquêté. Les entretiens réalisés par S. Pionchon tendent ainsi à montrer que fondamentalement, la socialisation reçue par les femmes les pousse moins souvent et moins fortement que les hommes à prendre gout aux joutes du pouvoir.

Entre soumission aux modèles socialement prescrits de réalisation de soi (épanouissement dans la vie familiale et la maternité) et «lucidité » propre aux dominés, non dupes de la vanité de la "comédie du pouvoir» (Françoise Giroud), cette distance des femmes vis-à-vis de la politique ne leur permet toujours pas d'investir le pouvoir au même titre que les hommes. Encore aujourd'hui, les femmes ne sont pas des «hommes» politiques comme les autres. 